\title{
The ELODIE survey for northern extra-solar planets
}

\section{HD 196885, a close binary star with a 3.7-year planet}

\author{
A. C. M. Correia ${ }^{1,2}$, S. Udry ${ }^{2}$, M. Mayor ${ }^{2}$, A. Eggenberger ${ }^{3,2}$, D. Naef ${ }^{4}$, J.-L. Beuzit ${ }^{3}$, C. Perrier $^{3}$, \\ D. Queloz ${ }^{2}$, J.-P. Sivan ${ }^{5}$, F. Pepe ${ }^{5}$, N. C. Santos ${ }^{6,2}$, and D. Ségransan ${ }^{5}$ \\ ${ }^{1}$ Departamento de Física da Universidade de Aveiro, Campus Universitário de Santiago, 3810-193 Aveiro, Portugal \\ e-mail: correia@ua.pt \\ 2 Observatoire de Genève, 51 Ch. des Maillettes, 1290 Sauverny, Switzerland \\ 3 Laboratoire d'Astrophysique de Grenoble, Université Joseph Fourier, BP 53, 38041 Grenoble Cedex 9, France \\ 4 European Southern Observatory, Casilla 19001, Santiago 19, Chile \\ 5 Laboratoire d'Astrophysique de Marseille, Traverse du Siphon BP 8, 13376 Marseille Cedex 12, France \\ ${ }^{6}$ Centro de Astrofísica da Universidade do Porto, Rua das Estrelas, 4150-762 Porto, Portugal
}

Received 23 October 2007 / Accepted 28 November 2007

\section{ABSTRACT}

\begin{abstract}
Aims. We aim to significantly increase the number of detected extra-solar planets in a magnitude-limited sample to improve our knowledge of their orbital element distributions and thus obtain better constraints for planet-formation models.

Methods. Radial-velocity data were taken at Haute-Provence Observatory (OHP, France) with the ELODIE echelle spectrograph. Results. We report the presence of a planet orbiting HD 196885 A, with an orbital period of 1349 days. This star was previously suggested to host a 386-day planet, but we cannot confirm its existence. We also detect the presence of a stellar companion, HD 196885 B, and give some constraints on its orbit.
\end{abstract}

Key words. stars: individual: HD 196885 A - stars: individual: HD 196885 B - stars: binaries: visual - stars: planetary systems techniques: radial velocities - methods: observational

\section{Introduction}

The ELODIE Planet Search Survey was an extensive radialvelocity northern survey of dwarf stars at the Haute-Provence Observatory (OHP, France) using the ELODIE high-precision fiber-fed echelle spectrograph (Baranne et al. 1996) mounted at the Cassegrain focus of the 1.93-m telescope. It started to operate at the end of 1993 and acquired data until the summer of 2006, when it was replaced by the new echelle spectrograph SOPHIE (Bouchy \& The Sophie Team 2006).

This survey is part of a large effort aimed at an extra-solar planet search through radial-velocity measurements, in order to characterize the types of exoplanets and to bring strong constraints on their processes of formation and evolution. ELODIE was responsible for finding many extra-solar planets, among them the first hot Jupiter, 51 Peg b (Mayor \& Queloz 1995). The original sample consisted of 142 stars, but a new sample of 330 stars was defined in 1997. Details of the program and the surveyed sample can be found in Perrier et al. (2003).

In this paper we present the detection of two bodies around the star HD $196885 \mathrm{~A}$, one in the mass range of planets and the other believed to be a stellar companion. This star was previously described to harbor a planet with a period of about 386 days (although no published reference is known), but we could not confirm its presence in our observations (our analysis shows a planet with an orbital period of about 1349 days instead). The stellar companion was also recently observed using NACO adaptative optics by Chauvin et al. (2006, 2007). The stellar properties of HD 196885 A are briefly recalled in Sect. 2 and the radial velocities with the two detected companions are described in Sect. 3. In Sect. 4 we discuss the possibility of the existence of more companions, in particular with orbital periods around 368 days. Last section is devoted to our conclusions.

\section{HD 196885 A stellar characteristics}

HD 196885 A was observed by the HIPPARCOS astrometric satellite (HIP 101966). A high-precision spectroscopic study of this star was also performed by Sousa et al. (2006) in order to examine the metallicity distribution of stars hosting planets. This star was also studied by near-infrared survey with adaptive optics of faint circumstellar environments, sensitive to companions within the stellar and the sub-stellar domains (Chauvin et al. 2006, 2007). Observed and inferred stellar parameters from these different sources are summarized in Table 1.

In the HIPPARCOS catalogue, HD $196885 \mathrm{~A}$ is given a spectral type F8 IV, a visual magnitude $V=6.398$ and a color index $B-V=0.559$. The measured parallax (30.31 \pm 0.81 mas) leads to a distance of $33.0 \pm 0.9 \mathrm{pc}$ and an absolute magnitude of $M_{V}=3.8 \pm 0.1$. From CORALIE spectra, Sousa et al. (2006) derived an effective temperature $T_{\text {eff }}=6340 \pm 39 \mathrm{~K}$, a gravity $\log g=4.46 \pm 0.02$ and a high metal content $[\mathrm{Fe} / \mathrm{H}]=0.29 \pm 0.05$ (Table 1). The bolometric correction $(B C=-0.006)$ is computed from Flower (1996) using the spectroscopic $T_{\text {eff }}$ determination. The bolometric magnitude is then $M_{\mathrm{Bol}}=3.791$, which allows us to derive a stellar luminosity of $L=2.40 L_{\odot}$. Sousa et al. (2006) also computed the mass, $M=1.33 M_{\odot}$, from evolutionary tracks using Geneva models (Schaller et al. 1992; Schaerer et al. 1993). From the $B-V$ value and ELODIE correlation functions we find $v \sin i=7.3 \pm 1.5\left[\mathrm{~km} \mathrm{~s}^{-1}\right]$, meaning that the star is rotating fast. However, its measured activity level 
Table 1. Observed and inferred stellar parameters for HD 196885 A.

\begin{tabular}{llc}
\hline \hline \multicolumn{1}{c}{ Parameter } & & HD 196885 A \\
\hline Spectral Type & & F8 V \\
$V$ & & 6.398 \\
$B-V$ & {$[\mathrm{mas}]$} & $0.559 \pm 0.006$ \\
$\pi$ & $30.31 \pm 0.81$ \\
$d$ & {$[\mathrm{pc}]$} & $33.0 \pm 0.9$ \\
$M_{V}$ & & $3.8 \pm 0.1$ \\
$B C$ & & -0.006 \\
$M_{\text {Bol }}$ & {$\left[L_{\odot}\right]$} & 3.79 \\
$L$ & & 2.40 \\
\hline$[\mathrm{Fe} / \mathrm{H}]$ & & $0.29 \pm 0.05$ \\
$\log R_{H K}^{\prime}$ & {$[\mathrm{days}]$} & -5.01 \\
$P_{\text {rot. }}$ & {$\left[M_{\odot}\right]$} & 15. \\
$M$ & {$[\mathrm{~K}]$} & 1.33 \\
$T_{\text {eff }}$ & {$[\mathrm{cgs}]$} & $6340 \pm 39$ \\
$\log g$ & {$\left[\mathrm{~km} \mathrm{~s}{ }^{-1}\right]$} & $4.46 \pm 0.02$ \\
$v \sin i$ & {$[\mathrm{Gyr}]$} & $7.3 \pm 1.5$ \\
age & $2.0 \pm 0.5$ \\
\hline
\end{tabular}

Photometric and spectral type are from Chauvin et al. (2006). Astrometric parameters are from HIPPARCOS (ESA 1997). The mass $M$ and the atmospheric parameters $T_{\text {eff }}, \log g$ and $[\mathrm{Fe} / \mathrm{H}]$ are from Sousa et al. (2006). The bolometric correction is computed from Flower (1996) using the spectroscopic $T_{\text {eff }}$ determination. The activity level and the rotation period are from Wright et al. (2004). The given age was obtained following the Bayesian approach of Pont \& Eyer (2004).

is very weak: $\log R_{H K}^{\prime}=-5.01$ (Wright et al. 2004). From this value, these authors inferred a rotation period of about 15 days. According to Pace \& Pasquini (2004), the activity level becomes weak and constant after $\sim 1.5 \mathrm{Gyr}$, setting a lower limit on the age of the star. On the other hand, following the Bayesian approach described in Pont \& Eyer (2004) we estimate a maximum age of $2.5 \mathrm{~Gy}$. The derived stellar atmospheric parameters and age are compatible with the expected values for a high metallicity late-F dwarf (F8 V), and are slightly at odds with the evolutionary status given by the HIPPARCOS catalogue.

\section{Orbital solutions for the HD 196885 system}

The ELODIE observations of HD 196885 A started in June 1997 and the last data acquired are from August 2006, since the ELODIE program was closed shortly after that date. The peculiar variations of the radial velocities (Fig. 1) and also non-confirmed announcements from other research teams (see Sect. 4), prevented us from announcing this system earlier. However, the recent detection of a visual small stellar companion close to the main star (Chauvin et al. 2006, 2007) confirmed our suspicion of a long term drift of the radial velocities. Superimposed on the drift we can also observe a regular variation of a few years signaling the presence of a sub-stellar companion.

Before the ELODIE program, the star HD 196885 A had been followed between June 1982 and August 1997 by the CORAVEL spectrometers (Baranne et al. 1979) mounted on the 1-m Swiss telescope at Haute-Provence Observatory and on the $1.54-\mathrm{m}$ Danish telescope at La Silla Observatory (ESO, Chile). The precision of CORAVEL is $\sim 0.3 \mathrm{~km} \mathrm{~s}^{-1}$, not enough to detect planetary objects, but very useful to help us to constrain the orbit of the stellar companion. From April 1999 to November 2002 a series of radial velocity measurements were also taken using the CORALIE echelle spectrograph (Queloz et al. 2000) mounted on the 1.2-m Swiss telescope at La Silla. This four year observational sequence is important to confirm the presence of the

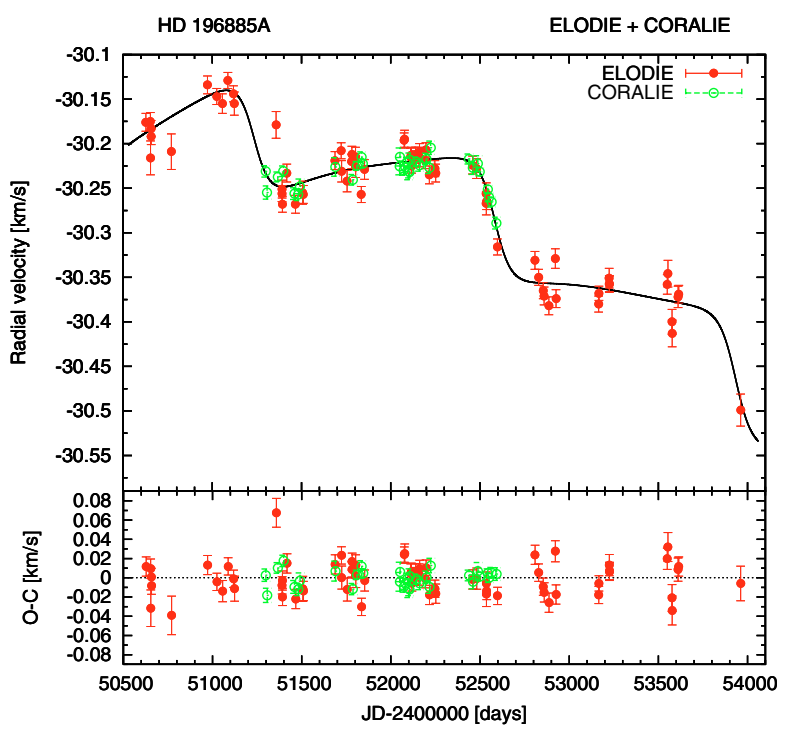

Fig. 1. ELODIE and CORALIE radial velocities for HD 196885 A, superimposed on a two-Keplerian orbital solution (Table 2).

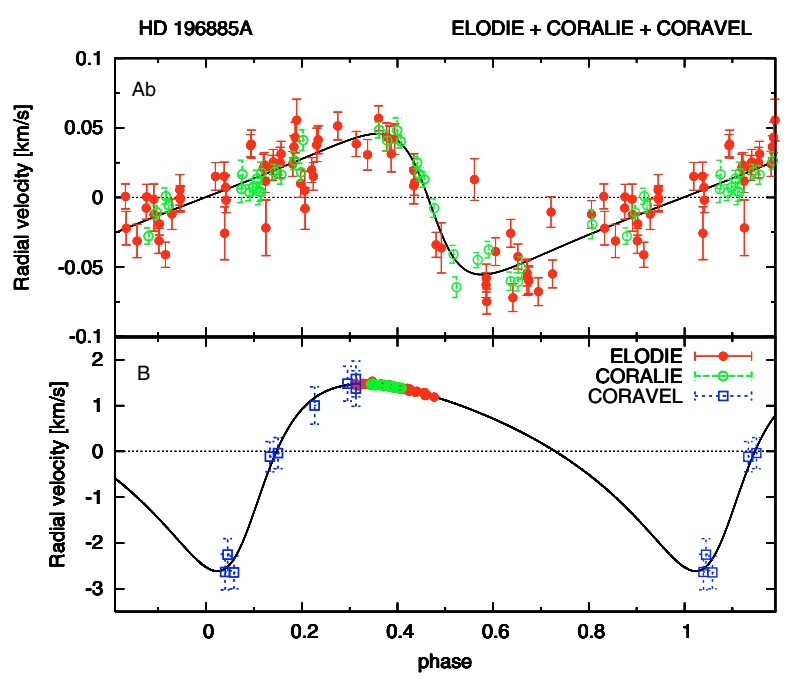

Fig. 2. Phase-folded radial velocities measurements and best fit for the planetary companion of HD 196885 A (top) and the its stellar companion (bottom). For each body the contribution by the other companion has been subtracted from the observational data. The orbital period of the inner body is $P=3.7 \mathrm{yr}$, while for the outer body we have $P=55 \mathrm{yr}$ (Table 2). However, the fact that we are unable to completely cover in phase the orbit of the stellar companion indicates that its orbital period may be considerably longer (Table 3 ).

planetary companion, since the precision of CORALIE is slightly better than the precision of ELODIE.

With 111 radial-velocity measurements (69 from ELODIE, 9 from CORAVEL and 33 from CORALIE), spanning $\sim 14$ years of observations, we are able to describe the orbit of the sub-stellar body in the system, as well as slightly constrain the orbit of the stellar companion. Using the iterative Levenberg-Marquardt method (Press et al. 1992), we first attempt to fit the complete set of radial velocities with a single orbiting companion and a quadratic drift. This fit yields a planetary companion with $P=1347$ days, $e=0.44$, a minimum mass of $2.9 M_{\text {Jup }}$ and an adjustment of $\sqrt{\chi^{2}}=1.811$ and $\mathrm{rms}=14.61 \mathrm{~m} / \mathrm{s}$. We then fit the radial velocities using a model with two Keplerian orbits (Figs. 1 and 2). It yields for 
Table 2. Orbital parameters for two bodies orbiting HD 196885 A, obtained with a two-Keplerian fit to observational data.

\begin{tabular}{llcc}
\hline \hline Param. & [unit] & HD 196885 Ab & HD 196885 B \\
\hline$V$ & {$\left[\mathrm{~km} \mathrm{~s}^{-1}\right]$} & \multicolumn{2}{c}{$-31.666 \pm 0.455$} \\
$P$ & {$[$ year] } & $3.69 \pm 0.03$ & $55.43 \pm 19.48$ \\
$e$ & & $0.462 \pm 0.026$ & $0.409 \pm 0.038$ \\
$\omega$ & {$[\mathrm{deg}]$} & $91.4 \pm 4.1$ & $227.6 \pm 23.4$ \\
$K$ & {$[\mathrm{~m} / \mathrm{s}]$} & $40.48 \pm 2.32$ & $2041 \pm 270$ \\
$T$ & {$[\mathrm{JD}-2400000]$} & $51236 \pm 18$ & $45928 \pm 2638$ \\
\hline$a_{1} \sin i$ & {$\left[10^{-3} \mathrm{AU}\right]$} & 4.45 & 3466 \\
$f(M)$ & {$\left[10^{-3} M_{\odot}\right]$} & $6.5 \times 10^{-6}$ & 13.56 \\
$M \sin i$ & {$\left[M_{\text {Jup }}\right]$} & 2.96 & 351.5 \\
$M \sin i$ & {$\left[M_{\odot}\right]$} & 0.0028 & 0.34 \\
$a$ & {$[\mathrm{AU}]$} & 2.63 & 17.23 \\
\hline $\mathrm{rms}$ & {$[\mathrm{m} / \mathrm{s}]$} & \multicolumn{3}{c}{11.87} \\
$\sqrt{\chi^{2}}$ & & \multicolumn{3}{c}{1.494} \\
\hline
\end{tabular}

Errors are given by the standard deviation $\sigma$. The orbital period of the outer body is much longer than the data acquired so far $(\sim 14 \mathrm{yr})$ and thus we are unable to completely constrain its orbit. However, we noticed that it is better to fit a complete elliptical orbit to the present data, rather than use a quadratic drift, for which we obtained $\sqrt{\chi^{2}}=1.811$ and $\mathrm{rms}=14.61 \mathrm{~m} / \mathrm{s}$. Alternative solutions for the outer body with longer orbital periods exist that match the data almost as well as the best fit (Table 3).

the inner planet $P=1349$ days, $e=0.46$ and a minimum mass of $3.0 M_{\text {Jup }}$, while for the outer companion we have $P \simeq 20000$ days, $e=0.41$ and a minimum mass of $0.34 M_{\odot}$ (Table 2). Despite all the uncertainties in the orbital parameters, the use of a Keplerian orbit for the massive outer body in the system proved to be a good approach, better than the quadratic drift, since the reduced $\sqrt{\chi^{2}}$ is now 1.494 and the velocity residuals drop to $\mathrm{rms}=11.87 \mathrm{~m} / \mathrm{s}$ (Fig. 2). Of course one can always argue that increasing by three the number of free parameters in the model will improve the fit, but at least we are able to place constraints for the orbital parameters of the outer body. For instance, we find that it must present an orbital period $P>40 \mathrm{yr}$, a semi-major axis $a>14 \mathrm{AU}$ and a minimum mass $M \sin i>0.28 M_{\odot}$, since all other fits coherent with the data found larger values for these parameters (Table 3 ).

As expected, the orbital parameters of the outer body still present some uncertainties around the best fitted value. This is particularly true for the orbital period, meaning that this parameter may assume rather different values. The LevenbergMarquardt method converges to a minimum $\chi^{2}$, but other close local minima also may represent a good fit to our data. For a particular choice of the orbital period, all the other parameters can adjust in a way such that the $\chi^{2}$ value remains close to the value from the best fit (Table 2). Thus, any orbital solution with a $\chi^{2}$ and an rms close to the values from Table 2 can be seen as a good model of the system (e.g. Table 3 ).

Chauvin et al. $(2006,2007)$ determined that HD 196885 is a close visual binary. They derive a possible mass for the companion of $0.5-0.6 M_{\odot}$ at a projected physical distance of $\sim 23 \mathrm{AU}$ and spectral type M1 V. As they noticed, for those values of the mass and semi-major axis we should be able to monitor its orbital motion using radial velocity measurements of the main star. In order to test if our drift can be interpreted as the observed stellar companion, we fitted our data by fixing orbital periods compared to those forecasted by Chauvin et al. (2007). As shown in Table 3, we are able to find multiple possibilities that match their observations. Notice also that the values of the mass listed
Table 3. Alternative orbital parameters for HD 196885 B.

\begin{tabular}{c|ccccc|cc}
\hline \hline $\begin{array}{c}P \\
{[\mathrm{yr}]}\end{array}$ & $\begin{array}{c}a \\
{[\mathrm{AU}]}\end{array}$ & $e$ & $\begin{array}{c}\omega \\
{[\mathrm{deg}]}\end{array}$ & $\begin{array}{c}T \\
{[\mathrm{JD}]}\end{array}$ & $\begin{array}{c}M \sin i \\
{\left[M_{\odot}\right]}\end{array}$ & $\begin{array}{c}\mathrm{rms} \\
{[\mathrm{m} / \mathrm{s}]}\end{array}$ & $\sqrt{\chi^{2}}$ \\
\hline 40 & 13.7 & 0.50 & 204 & 45983 & 0.28 & 11.95 & 1.504 \\
60 & 18.3 & 0.41 & 233 & 45916 & 0.36 & 11.87 & 1.494 \\
80 & 22.4 & 0.42 & 250 & 46136 & 0.43 & 11.88 & 1.495 \\
100 & 26.6 & 0.45 & 258 & 45987 & 0.55 & 11.88 & 1.495 \\
120 & 30.4 & 0.49 & 265 & 46110 & 0.61 & 11.89 & 1.496 \\
\hline
\end{tabular}

With the present $\sim 14$ years of observational data it is still impossible to completely constrain the outer body orbit. Observations by Chauvin et al. (2007) point to a small stellar companion with $0.5-0.6 M_{\odot}$ at about 23 AU. Fixing the orbital period at larger values, we are able to find orbital solutions compatible with those observations.

in Table 3 correspond to minimum masses. Because of the factor $\sin i$, the real masses can be larger. We then conclude that the outer companion of the star HD $196885 \mathrm{~A}$ is almost certainly the stellar mass object observed by Chauvin et al. (2006, 2007), denoted HD 196885 B. The inner companion of HD 196885 A remains a planetary body, hereafter called HD $196885 \mathrm{Ab}$, with orbital parameters listed in Table 2.

Finally, we note that the HD 196885 system may be a part of a wider binary with the star BD+104251 B located 191.9" North of HD 196885 A (Trilling et al. 2007). At a distance of 33 pc measured by Perryman et al. (1997), we estimate a semi-major axis of $6330 \mathrm{AU}$, that is, a drift contribution of $\sim 1 \mathrm{~cm} \mathrm{~s}^{-1} \mathrm{yr}^{-1}$ to the radial velocity of HD $196885 \mathrm{~A}$ (assuming a circular orbit), and thus impossible to be detected with ELODIE.

\section{A second planet around one year?}

In 2004, a $P=386$ days companion to the HD 196885 A star was reported by the California \& Carnegie Planet Search Team on their webpage ${ }^{1}$, detected through radial-velocity measurements obtained with the Lick survey. This planet was also listed in the exoplanets Encyclopedia ${ }^{2}$, by Fischer \& Valenti (2005), Chauvin et al. (2007) and Marchi (2007). Unfortunately no publication describing the system is known, as noticed by many other authors (Chauvin et al. 2006; Sousa et al. 2006; Bonavita \& Desidera 2007; Desidera \& Barbieri 2007). Moreover, the system is not reported in the 2006 exoplanet catalog by Butler et al. (2006), so we assume there was no confirmation of this planet after the first announcement.

Curiously, performing a frequency analysis of the ELODIE radial velocity residuals (Fig. 1), we find an important peak signature at about $P=368$ days (Fig. 3 ), that could be interpreted as a second planet around HD 196885 A. However, this peak is not present when we analyze CORALIE data, casting some doubts on its origin. Computing the ELODIE window function, we also note an important peak at exactly 368 days (Fig. 3), clearly suggesting that the peak shown in the frequency analysis is an aliasing of the observational data. Performing a Keplerian fit to the ELODIE residuals, we find that the best fit corresponds to solutions with very high eccentricities $(e \sim 0.8)$, that are not dynamical stable. Moreover, when we plot a phase-fold of the residual radial velocities (Fig. 4), our observations only cover half of the orbit, preventing us from fully constraining it. Finally, we performed a Keplerian fit of the three bodies also including data

\footnotetext{
${ }^{1}$ http://exoplanets.org/esp/hd196885/hd196885.shtml

${ }^{2}$ http://exoplanet.eu/planet.php?p1=HD+196885\&p2=b
} 


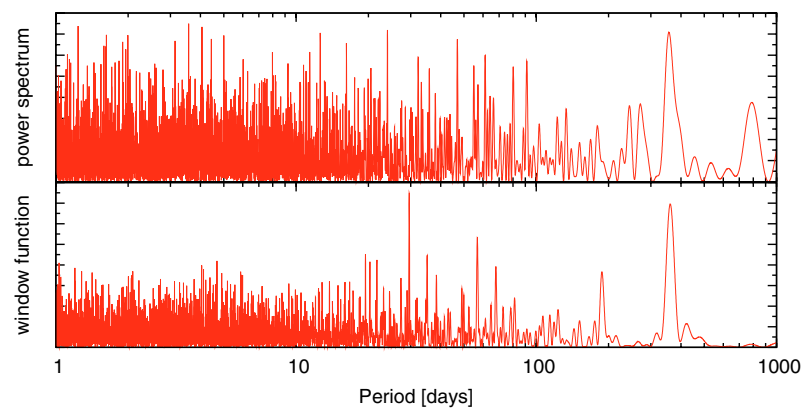

Fig. 3. Frequency analysis (top) and window function (bottom) for ELODIE residual radial velocities of HD 196885 A when the contributions from the two bodies fitted in Table 2 are subtracted. An important peak is detected at $P=368$ days, which could be interpreted as a third body in the system. However, looking at the window function, we see that the same peak is present, indicating that this may be an artifact.
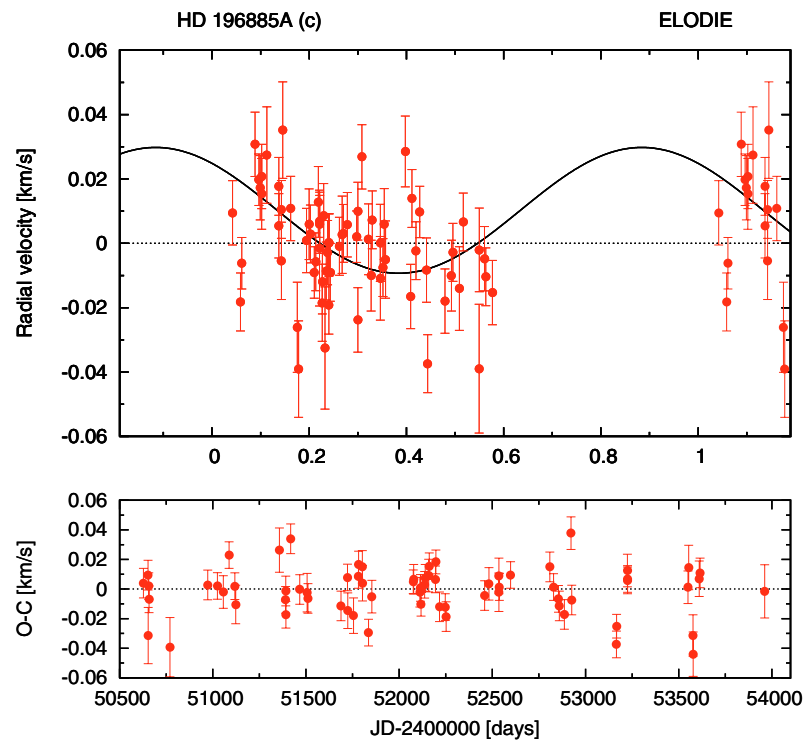

Fig. 4. Phase-folded ELODIE residual radial velocities for HD 196885 A when the contributions from the two bodies fitted in Table 2 are subtracted. Data are superimposed on a Keplerian solution with $e=0$ and $P=368$ days. The respective residuals as a function of Julian Date are displayed in the lower panel. We see that ELODIE data only cover half of the orbital phase, preventing us from completely constraining it. Moreover, the inclusion of this third body does not improve the fit significantly. We then conclude that the presence of a third body at $P=368$ days is an artifact.

from CORALIE and CORAVEL. We found $\sqrt{\chi^{2}}=1.460$ and $\mathrm{rms}=11.30 \mathrm{~m} / \mathrm{s}$, which does not represent a substantial improvement with respect to the system with only two companions (Table 2). The presence of a companion around 368 days can thus be discarded.

Besides the artifactual companion at 368 days, we may ask if there are other companions at different orbital periods. For that purpose we used a genetic algorithm, since we cannot clearly isolate any other peak in the frequency analysis of Fig. 3. The inclusion of a third companion in the system allows us to reduce the $\sqrt{\chi^{2}}$ to 1.25 and the $\mathrm{rms}=10.5 \mathrm{~m} / \mathrm{s}$. This represents only a slightly better adjustment of the model to the data that can be justified as a natural consequence of increasing the number of free parameters. Moreover, identical adjustments can be obtained with many orbital periods, as different as 1.3 days and 10 days, frequently with very high eccentricity values. Therefore, no other companion can be conclusively detected in the residuals from the orbital solution listed in Table 2.

\section{Discussion and conclusion}

In this paper we report the presence of a planet orbiting the HD 196885 A star, with an orbital period of 1349 days. We also detect the presence of the stellar companion HD $196885 \mathrm{~B}$, that was recently observed by Chauvin et al. (2006, 2007). Its orbit is not completely determined, but we are able to provide some constraints: $P>40 \mathrm{yr}, a>14 \mathrm{AU}$ and $M \sin i>0.28 M_{\odot}$. It is possible to find a large set of orbits with much longer orbital periods that fit the observational data as well as the best fit (Table 3). In particular, we find a solution at $P=120 \mathrm{yr}$ with $M \sin i=0.6 M_{\odot}$, the mass estimated by Chauvin et al. (2007).

The HD 196885 A system was previously described as a star orbited by a planet at 386 days by the California \& Carnegie Planet Search Team, detected with data acquired with the Lick survey. Although the system was reported on their webpage and many other places, no publication describing the system is known. The data acquired with the ELODIE survey also present some signal around 368 days, but a more detailed analysis shows that this signal corresponds to an aliasing of the observational data, probably due to the similarities with the Earth orbital period. We thus do not confirm the existence of the planet obtained with measurements form the Lick survey.

The ELODIE radial velocity residuals after subtracting the signal from the two companions still presents an $\mathrm{O}-\mathrm{C}$ (Fig. 1) slightly above the precision of the instrument. This suggests that the system may still hide additional planetary companions. However, we were unable to find them, even when using a genetic algorithm. The excess in the residuals can also be a contamination from the spectrum of the stellar companion HD 196885 B. We have searched for its presence in our ELODIE data using the multi-order TODCOR, a two-dimensional crosscorrelation algorithm (Zucker et al. 2004), but did not find anything convincing. We have also searched for correlations between line bisectors and radial velocity or residuals. No significant correlation could be detected. These negative results do not allow us to formally discard the blend scenario, but they render it an unlikely possibility.

The planet HD $196885 \mathrm{Ab}$ is the fourth to be discovered in a close binary with a separation smaller than $25 \mathrm{AU}$. The other cases already known are Gliese 86 (Queloz et al. 2000; Lagrange et al. 2006), $\gamma$ Cep (Hatzes et al. 2003; Neuhäuser et al. 2007) and HD 41004 A (Zucker et al. 2004). These systems are ideal for carrying out combined astrometric and radial-velocity observations to constrain the binary dynamic properties and the possible impact of a close binary companion on planet formation and evolution.

Acknowledgements. We acknowledge support from the Swiss National Research Found (FNRS), the Geneva University and French CNRS. A.C. and N.S. benefited from Portuguese FCT grant POCI/CTE-AST/56453/2004 and N.S. was also supported from the EC's FP6 and by FCT (with POCI2010 and FEDER funds), within the HELAS international collaboration. A.E. acknowledges support from the Swiss National Science Foundation through a fellowship for a prospective researcher. We are grateful to the Observatoire de HauteProvence for the generous time allocation.

Note: After the submission of the present paper the web sites were updated with the orbital solution described here. Moreover, the Lick Planet Search Team also agree with this new solution for HD 196885 (Debra Fisher, private communication). 


\section{References}

Baranne, A., Mayor, M., \& Poncet, J. L. 1979, Vistas Astron., 23, 279 Baranne, A., Queloz, D., Mayor, M., et al. 1996, A\&AS, 119, 373

Bonavita, M., \& Desidera, S. 2007, A\&A, 468, 721

Bouchy, F., \& The Sophie Team. 2006, in Tenth Anniversary of 51 Peg-b: Status of and prospects for hot Jupiter studies, ed. L. Arnold, F. Bouchy, \& C. Moutou, 319

Butler, R. P., Wright, J. T., Marcy, G. W., et al. 2006, ApJ, 646, 505

Chauvin, G., Lagrange, A.-M., Udry, S., et al. 2006, A\&A, 456, 1165

Chauvin, G., Lagrange, A. M., Udry, S., \& Mayor, M. 2007, A\&A, 475, 723

Desidera, S., \& Barbieri, M. 2007, A\&A, 462, 345

ESA. 1997, The Hipparcos and Tycho Catalogues, 1239

Fischer, D. A., \& Valenti, J. 2005, ApJ, 622, 1102

Flower, P. J. 1996, ApJ, 469, 355

Hatzes, A. P., Cochran, W. D., Endl, M., et al. 2003, ApJ, 599, 1383

Lagrange, A.-M., Beust, H., Udry, S., Chauvin, G., \& Mayor, M. 2006, A\&A, 459,955

Marchi, S. 2007, ApJ, 666, 475
Mayor, M., \& Queloz, D. 1995, Nature, 378, 355

Neuhäuser, R., Mugrauer, M., Fukagawa, M., Torres, G., \& Schmidt, T. 2007, A\&A, 462, 777

Pace, G., \& Pasquini, L. 2004, A\&A, 426, 1021

Perrier, C., Sivan, J.-P., Naef, D., et al. 2003, A\&A, 410, 1039

Perryman, M. A. C., Lindegren, L., Kovalevsky, J., et al. 1997, A\&A, 323, L49

Pont, F., \& Eyer, L. 2004, MNRAS, 351, 487

Press, W. H., Teukolsky, S. A., Vetterling, W. T., \& Flannery, B. P. 1992, Numerical recipes in FORTRAN. The art of scientific computing (Cambridge: University Press, 2nd ed.)

Queloz, D., Mayor, M., Weber, L., et al. 2000, A\&A, 354, 99

Schaerer, D., Meynet, G., Maeder, A., \& Schaller, G. 1993, A\&AS, 98, 523

Schaller, G., Schaerer, D., Meynet, G., \& Maeder, A. 1992, A\&AS, 96, 269

Sousa, S. G., Santos, N. C., Israelian, G., Mayor, M., \& Monteiro, M. J. P. F. G. 2006, A\&A, 458, 873

Trilling, D. E., Stansberry, J. A., Stapelfeldt, K. R., et al. 2007, ApJ, 658, 1289

Wright, J. T., Marcy, G. W., Butler, R. P., \& Vogt, S. S. 2004, ApJS, 152, 261

Zucker, S., Mazeh, T., Santos, N. C., Udry, S., \& Mayor, M. 2004, A\&A, 426, 695 